\title{
Expression of EMT markers and mode of surgery are prognostic in phyllodes tumors of the breast
}

\author{
Xiaolong Feng ${ }^{1}$, Lin Zhao ${ }^{1}$, Honghong Shen ${ }^{1}$, Xiaozhen Liu ${ }^{1}$, Yang Yang ${ }^{1}$, Shuhua Lv \\ and Yun Niu ${ }^{1}$ \\ ${ }^{1}$ Key Laboratory of Breast Cancer Prevention and Therapy (Ministry of Education), Tianjin Medical University Cancer Institute \\ and Hospital, National Clinical Research Center for Cancer, Key Laboratory of Cancer Prevention and Therapy, Tianjin, China \\ ${ }^{2}$ Department of Pathology, Tianjin Union Medical Center, Tianjin People's Hospital, Tianjin, China \\ Correspondence to: Yun Niu, email: yunniu2015@126.com \\ Keywords: phyllodes tumors of the breast, epithelial to mesenchymal, mesenchymal stem cells, clinicopathological features, \\ survival rate \\ Received: September 14, $2016 \quad$ Accepted: February 27, $2017 \quad$ Published: March 23, 2017 \\ Copyright: Feng et al. This is an open-access article distributed under the terms of the Creative Commons Attribution License \\ (CC-BY), which permits unrestricted use, distribution, and reproduction in any medium, provided the original author and source \\ are credited.
}

\section{ABSTRACT}

Phyllodes tumors of the breast are rare neoplasms that account for $<1 \%$ of all mammary tumors and 2-3\% of fibro-epithelial neoplasms of the breast. We evaluated the clinicopathological characteristics of a cohort of 246 Chinese patients in relation to the expression of epithelial-to-mesenchymal (EMT) markers in benign, borderline and malignant tumors and the prognostic value of different surgical regimens. We observed that survival outcomes correlated with the mode of surgical management in the three patient groups. Expression of E-cadherin, Snail, Slug and Twist were higher in epithelial cells from borderline and malignant tumors than those in benign tumors, whereas the expression of $\mathbf{N}$-cadherin was opposite. Levels of the EMT markers Snail and Slug in the stromal compartment increased with the advancing tumor grade. Expression of mesenchymal stem cell markers contributed to the inherent heterogeneity in the malignant tumors. Based on Cox models, surgical management emerged as an independent predictor for disease-free survival, whereas a history of recent growth and tumor grade were independent predictors for overall survival. These findings show that expression of EMT markers, the mode of surgical management, and a history of recent tumor growth had prognostic potential for patients with phyllodes tumors of the breast.

\section{INTRODUCTION}

Phyllodes tumors (PT) of the breast are rare bidirectional neoplasms that represent $<1 \%$ of all mammary tumors and $2 \%$ to $3 \%$ of fibroepithelial neoplasms of the breast and are typified by stromal proliferation [1-4]. The stromal changes are considered key during PT progression and the differences between the three stages of PTs has been widely debated over the last century. In 1838, Müller described the first case and called it "cystosarcoma phyllodes of the breast" [5]. More than half a century later, the term "cystosarcoma phyllodes" was replaced by "phyllodes tumor" to distinguish it from purely mesenchymal malignant tumors. During the 1970s, PT were classified into two categories, benign and malignant. In 1982, the
World Health Organization adopted a new histopathologic classification of PT that included three categories, namely, benign, borderline, and malignant [6]. Although the histopathological type (benign, borderline or malignant) was an independent prognostic factor for survival, several other histological parameters that were prognostic factors in breast carcinomas were uncertain for PT. Further, although surgery is the standard treatment for PTs, the best type of surgery (local excision, breast-conserving surgery, total mastectomy or modified/radical mastectomy) has not been determined $[1,2,7]$. In the benign form of PT, wide local excision is usually preferred after taking the breast volume and tumor size into account. Total mastectomy (TM) is reserved to treat local recurrence (LR). However, the role of conservative surgery has not been clearly defined for borderline and 
malignant tumors. Also, the prognostic factors relevant to derive treatment options were limited by the low numbers of specimens available for studies.

The epithelial-mesenchymal transition (EMT) is a biological transformation process that enables transforming cells to acquire mesenchymal functions that include the ability of migration, invasiveness and elevated resistance to apoptosis [8]. Many studies regarding EMT have identified biomarkers for epithelial and mesenchymal cells such as E-cadherin, Vimentin, Snail, Twist and Slug [9-18]. All the grades of PTs showed local recurrence with the borderline and malignant forms showing the potential to metastasize to organs such as the lungs, bone and the liver. Therefore, the focus regarding metastasis of malignant PTs requires recognizing the stemness within the primary PTs similar to liposarcoma, leiomyosarcoma, rhabdomyosarcoma, osteogenic sarcoma or chondrosarcoma [19]. The multifunctionality of PTs demonstrating diverse sarcoma types is reminiscent of the mesenchymal stem cells (MSCs). Some studies showed that malignant PTs possess MSC-like properties and the mesenchymal biomarkers such as $\mathrm{GD}_{2}$ and $\mathrm{ALDH}_{1}$ that are highly expressed in mesenchymal stem cells [19]. Therefore, in this study of 246 patients, we explored the expression of EMT and MSCs biomarkers in different grades of PT (benign, borderline or malignant) with a goal to identify relevant prognostic factors and therapeutic options for benign, borderline and malignant PTs.

\section{RESULTS}

\section{Clinicopathological parameters of patients}

The clinical parameters of the 246 PT cases are summarized in Table 1. The 246 patients cases were subdivided into three categories based on the histological type at diagnosis, namely, $42.3 \%$ benign (104 cases), $44.3 \%$ borderline (109 cases) and 13.4\% malignant (33 cases). The 33 malignant tumor cases included five that were poorly differentiated with heterologous metaplasia. The age of the patients ranged from 11 to 76 years old (median: 46 years) at diagnosis. The median diameter of all phyllodes tumors was $6.0 \mathrm{~cm}$ (range: $1.0-30 \mathrm{~cm}$ ). The median diameter was $4.5 \mathrm{~cm}$ (range: $1.3-30 \mathrm{~cm}$ ) for benign tumors, $4.5 \mathrm{~cm}$ (range: $1.0-28 \mathrm{~cm}$ ) for borderline tumors and $5 \mathrm{~cm}$ (range: $1.5-18 \mathrm{~cm}$ ) for malignant tumors. At diagnosis, $77(31.3 \%)$ patients were menopausal. Tumors in $145(59 \%)$ patients were located in the left breast. 56 $(23 \%)$ patients had recent tumor growth. $50(20 \%)$ patients had experienced painful symptoms associated with the development of the disease. There was no statistical difference in age at surgery, tumor diameter, menopausal status, recent growth or painful symptoms between the three groups $(p=0.498, p=0.345, p=0.903, p=0.706$ and $p=0.346$, respectively). Of the 246 patients, $11(4.5 \%)$ received breast-conserving surgery, 136 (55.3\%) were treated by lumpectomy, $29(11.8 \%)$ were treated by mastectomy and $70(28.5 \%)$ were treated by modified or radical mastectomy. Significant differences existed between three groups according to different surgical managements (breast-conserving surgery, lumpectomy, mastectomy or modified/ radical mastectomy; $\mathrm{P}<0.001$ ).

\section{Immunohistochemistry of EMT biomarkers}

Expression of EMT markers was assessed using immunohistochemistry (IHC) in the 246 cases. Four cases (3 malignant PTs and one borderline case) were excluded from this study due to poor quality of the stained tissue section. IHC staining was separately scored for epithelial and stromal cells based on staining intensity and the percentage of positive cells. Stromal overgrowth was defined as absence of an epithelial component in at least one microscopic field at $40 \times$ total magnification $(10 \times$ ocular objective and $4 \times$ microscopic lens objective). Excessive proliferation of stromal cells was a feature of borderline and malignant PTs. As shown in Figure 1, EMT markers expressed in at least $5 \%$ of cells were recognized as positive [19]. EMT markers were detected in the epithelial cells of 104 benign PTs with the following frequencies: E-cadherin (64.4\%, 67/104), N-cadherin (45.2\%, 47/104), Snail (34.6\%, 36/104), Slug (31.7\%, 33/104), Twist $(34.6 \%, 36 / 104)$. Likewise, for the borderline $(n=108)$ and malignant $(\mathrm{n}=30)$ PTs, the frequencies obtained were as follows: E-cadherin $(48.1 \%, 52 / 108)$ and $(43.3 \%$, $13 / 30)$, N-cadherin $(57.4 \%, 62 / 108)$ and $(43.3 \%, 13 / 30)$, Snail $(52.8 \%, 57 / 108)$ and $(70.0 \%, 21 / 30)$, Slug (63.0\%, $68 / 108)$ and $(73.3 \%, 22 / 30)$, Twist $(41.7 \%, 45 / 108)$ and $(60.0 \%, 18 / 30)$. Whereas, the expression of E-cadherin, Snail, Slug and Twist in epithelial cells of benign PTs was lower than the borderline and malignant PTs, $(P=0.025$, $P=0.001, P<0.001, P=0.044$, respectively), no difference was observed for $\mathrm{N}$-cadherin $(P=0.146)$. For the EMT markers highlighting the positive stroma component, Snail $(P=0.007)$ and Slug $(P=0.001)$ increased with the advancing grade of PTs (Table 2 ).

\section{Expression of mesenchymal stem cells (MSCs) markers in malignant PTs}

To verify co-expression of MSC and EMT markers, 20 malignant cases were randomly picked for immunofluorescence. Co-expression of $\mathrm{MSC}\left(\mathrm{GD}_{2}\right.$ and $\mathrm{ALDH}_{1}$ ) and EMT markers was observed in 15 cases. Also, nuclear (Slug and Twist) and cytoplasmic $\left(\mathrm{GD}_{2}\right.$ and $\left.\mathrm{ALDH}_{1}\right)$ staining was observed in these malignant PTs (Figure 2).

\section{Follow-up and survival analysis}

The median duration of follow-up was 102.5 months (range 60-345). During this time, 45 patients (18.3\%) experienced recurrence and/or metastasis and 
Table 1: Differences between tumor grade and clinicopathological parameters in 246 patients with phyllodes tumor of the breast (Values of $\mathbf{P}<0.05$ were considered significant)

\begin{tabular}{|c|c|c|c|c|c|}
\hline Parameters & Benign & Borderline & Malignant & $\chi^{2}$ & $P$ values \\
\hline \multicolumn{6}{|l|}{ Age at surgery (years) } \\
\hline$\leq 50$ & $73(70.2 \%)$ & $70(64.2 \%)$ & $20(60.6 \%)$ & \multirow{2}{*}{1.4} & \multirow{2}{*}{0.498} \\
\hline$>50$ & $31(29.8 \%)$ & $39(35.8 \%)$ & $13(39.4 \%)$ & & \\
\hline \multicolumn{6}{|l|}{ Tumor size (cm) } \\
\hline$\leq 3$ & $29(27.9 \%)$ & $34(31.2 \%)$ & $6(18.2 \%)$ & \multirow{2}{*}{2.1} & \multirow{2}{*}{0.345} \\
\hline$>3$ & $75(72.1 \%)$ & $75(68.8 \%)$ & $27(81.8 \%)$ & & \\
\hline \multicolumn{6}{|l|}{ Menopausal status } \\
\hline Yes & $31(29.8 \%)$ & $35(32.1 \%)$ & $11(33.3 \%)$ & \multirow{2}{*}{0.2} & \multirow{2}{*}{0.903} \\
\hline No & $73(70.2 \%)$ & $74(67.9 \%)$ & $22(66.7 \%)$ & & \\
\hline \multicolumn{6}{|l|}{ Recent growth } \\
\hline Yes & $22(21.2 \%)$ & $28(25.7 \%)$ & $7(21.2 \%)$ & \multirow{2}{*}{0.7} & \multirow{2}{*}{0.706} \\
\hline No & $82(78.8 \%)$ & $81(74.3 \%)$ & $26(78.8 \%)$ & & \\
\hline \multicolumn{6}{|l|}{ Painful symptoms } \\
\hline Yes & $20(19.2 \%)$ & $21(19.3 \%)$ & $10(30.3 \%)$ & \multirow{2}{*}{2.1} & \multirow{2}{*}{0.346} \\
\hline No & $84(80.8 \%)$ & $88(80.7 \%)$ & $23(69.7 \%)$ & & \\
\hline \multicolumn{6}{|l|}{ Surgical management* } \\
\hline Breast-conserving surgery & $5(4.8 \%)$ & $6(5.5 \%)$ & $0(0.0 \%)$ & \multirow{4}{*}{49.3} & \multirow{4}{*}{$<0.001$} \\
\hline Lumpectomy & $70(67.3 \%)$ & $62(56.9 \%)$ & $4(12.1 \%)$ & & \\
\hline Mastectomy & $14(13.5 \%)$ & $11(10.1 \%)$ & $4(12.1 \%)$ & & \\
\hline Modified/radical mastectomy & $15(14.4 \%)$ & $30(27.5 \%)$ & $25(75.8 \%)$ & & \\
\hline
\end{tabular}

$P$ values were calculated by Chi-square test.

* There were significant differences among benign, borderline and malignant phyllodes tumor of the breast in surgical management $\left(\chi^{2}=49.3, P<0.001\right)$.

25 patients (10.2\%) died. The most common focus of recurrence was the tumor bed and the most common metastasis was lung, followed by liver and brain. The rate of recurrence and metastasis in the malignant PTs group was $42.4 \%$ (14/33) compared to the borderline and benign groups that showed $26.6 \%(29 / 109), 13.5 \%$ $(14 / 104)$, respectively $(P=0.001)$. Similarly, the overall survival was different among the three groups $(P<0.01)$. Overall, the cohort of patients showed a 5-year DFS of $82.9 \%$ and a 5 -year OS of $92.3 \%$. The 5 -year OS for the benign, borderline and the malignant groups was $97.1 \%(101 / 104), 96.3 \%(105 / 109)$ and $78.8 \%(26 / 33)$, respectively $(P<0.001)$. The 5 -year DFS for the three groups was $95.2 \%$ (99/104), 80.7\% (88/109), 66.7\% $(22 / 33)$, respectively and was statistically significant $(P<0.001$; Table 3$)$. Based on the Kaplan-Meier and logrank statistic methods, we observed that the 5-year DFS and 5-year OS was significantly lower in the malignant group in comparison to the borderline and benign groups (Figure 3).

Based on univariate analysis (Table 4), advanced grade $(P=0.01)$, Slug overexpression $(P=$ $0.036)$ and surgical management $(P<0.01)$ of PTs were the significant predictive factors for DFS. Only surgical management $(P=0.009)$ was significant in the multivariate analysis. For OS, advanced grade at diagnosis $(P<0.01)$, history of recent growth $(P=0.007)$, Slug overexpression $(P=0.028)$ and surgical management $(P=0.002)$ of tumors were the significant predictive factors in univariate analysis, whereas, history of recent growth and grade of PTs were significant predictive factors in multivariate analysis $(P=0.003$ and $0.001)$. The rest of the parameters like age, tumor size, menopausal state, painful symptoms and expression of EMT markers (E-cadherin, N-cadherin, Snail, and Twist) were not statistically significant. 


\section{DISCUSSION}

Phyllodes tumors are uncommon fibroepithelial tumors of breast that are difficult to pre-operatively diagnose with unpredictable clinical outcomes [6]. Although the biomarkers of PTs have been investigated previously, reliable biomarkers for clinical diagnosis and adjuvant therapies remain unclear $[20,21]$. The invasive and metastatic stages of breast cancer progression are closely linked to the epithelial-mesenchymal transition (EMT) that results in the transformed cells acquiring the abilities of invasiveness, migratory and stemness [11, 22-24]. In our study, we hypothesized that the EMT process was associated with PTs progression and therefore analyzed the status of the EMT markers in different stages of the tumors in the 246 clinical specimens. We demonstrated overexpression of key EMT proteins, namely, E-cadherin, N-cadherin, Snail, Slug and Twist in PTs. We also observed that increased E-cadherin, Snail, Slug and Twist expression was associated with the malignant phenotype of PTs. Our findings were concordant with previous studies that showed that EMT markers (E-cadherin, Snail, Slug and Twist) expression $(P=0.025$, $P=0.001, P<0.01, P=0.044$, respectively) correlated more strongly with borderline and malignant PTs than with benign PT [25]. However, no significant difference in $\mathrm{N}$-cadherin $(P=0.146)$ expression was observed between the malignant/borderline and the benign groups. Similarly, in stromal cells, Snail $(P=0.07)$ and Slug $(P=0.01)$ overexpression correlated with the increasing grades of PTs.

The clinicopathological features of the malignant PT patients showed the largest rate of modified or radical mastectomy. In spite of extensive resection, the malignant patients had most local recurrences and/or metastasis accounting for the worst five-year DFS and OS. It was observed that the grade of PTs influenced their distinct biological behaviour [26]. Tse and colleagues showed that the rate of recurrence increased as the grades progressed and ranged from 10 to $25 \%$ for benign to $32 \%$ for borderline and about $40 \%$ for malignant cases [26]. In our study, there was a $13.5 \%, 26.6 \%$ and $42.4 \%$ local recurrence and/or metastasis in the three groups with longer follow-up. As expected, the malignant group had poorer prognosis than the borderline and benign groups. Compared to malignant epithelial neoplasm of breast and true sarcomatous tumors, the malignant phyllodes tumors were not as lethal. A former epidemiologic review showed that malignant PTs had a 5-year survival rate of $80 \%$ after surgery [27]. Our results were consistent with the previous reports with a 5-year-OS of $78.8 \%$ and 5-yearDFS of $66.7 \%$. Clinicopathological factors like the grade of tumors, mode of surgery and Slug expression influenced patient survival.

We further evaluated expression of EMT markers in epithelial and stromal cells of the PTs specimens. We
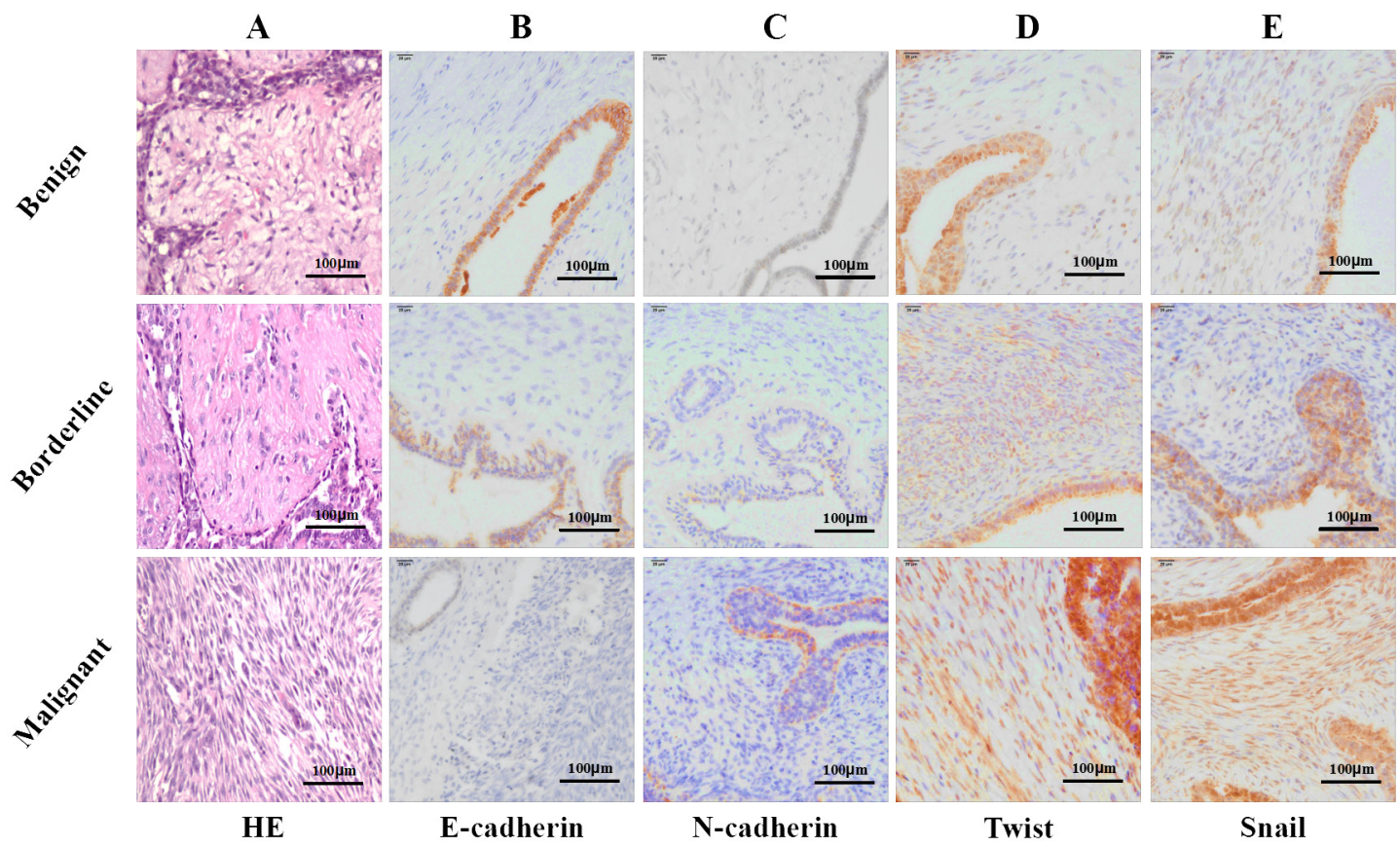

Figure 1: Representative photomicrographs of hematoxylin and eosin stain $(H \& E)$ and immunohistochemical stain (IHC) for E-cadherin, N-cadherin, Twist and Snail. (A) H\&E stained benign, borderline and malignant phyllodes tumor specimens (magnification $\times 200$ ); (B) IHC showing E-cadherin expression in epithelial and stromal compartment ( magnification $\times 400$ ); (C) IHC showing N-cadherin expression in epithelial and stroma compartment (magnification $\times 400$ ); (D) IHC showing Twist expression in epithelial and stroma component (magnification $\times 400$ ); (E) IHC showing Snail expression in epithelial and stroma component (magnification $\times 400$ ). 
Table 2: Differences between tumor grade and EMT markers in phyllodes tumor of the breast (Values of $\mathbf{P}<0.05$ were considered significant)

\begin{tabular}{lccccc}
\hline Parameters & Benign & Borderline & Malignant & $\chi^{2}$ & $P$ values \\
\hline $\begin{array}{l}\text { E-cadherin } \\
\text { epithelial * }\end{array}$ & $67(64.4 \%)$ & $52(48.1 \%)$ & $13(43.3 \%)$ & 7.4 & 0.025 \\
stroma & $33(31.7 \%)$ & $32(29.6 \%)$ & $10(33.3 \%)$ & 0.2 & 0.906 \\
$\begin{array}{c}\text { N-cadherin } \\
\text { epithelial }\end{array}$ & & & & \\
$\quad$ stroma & $47(45.2 \%)$ & $62(57.4 \%)$ & $13(43.3 \%)$ & 3.9 & 0.146 \\
$\begin{array}{l}\text { Snail } \\
\text { epithelial* }\end{array}$ & $35(33.7 \%)$ & $48(44.4 \%)$ & $17(56.7 \%)$ & 5.9 & 0.053 \\
stroma** & $36(34.6 \%)$ & $57(52.8 \%)$ & $21(70.0 \%)$ & 14.2 & 0.001 \\
Slug & $35(33.7 \%)$ & $52(48.1 \%)$ & $19(63.3 \%)$ & 9.8 & 0.007 \\
epithelial* & & & & & \\
stroma** & $33(31.7 \%)$ & $68(63.0 \%)$ & $22(73.3 \%)$ & 27.6 & $<0.001$ \\
Twist & $33(31.7 \%)$ & $58(53.7 \%)$ & $19(63.3 \%)$ & 14.7 & 0.001 \\
$\quad$ epithelial* & & & & & \\
stroma & $36(34.6 \%)$ & $45(41.7 \%)$ & $18(60.0 \%)$ & 6.3 & 0.044 \\
\hline
\end{tabular}

$P$ values were calculated by Chi-square test.

" Immunohistochemical staining for EMT markers in the epithelial was analyzed by Chi-square test. There were significant differences among benign, borderline and malignant phyllodes tumor of the breast in E-cadherin, Snail, Slug and Twistpositive of epithelial cells $\left(\chi^{2}=7.4, P=0.025 ; \chi^{2}=14.2, P=0.001 ; \chi^{2}=27.6, P<0.001 ; \chi^{2}=6.3, P=0.044\right)$.

${ }^{* *}$ Immunohistochemical staining for EMT markers in the stroma was analyzed by Chi-square test. There were significant differences among benign, borderline and malignant phyllodes tumor of the breast in Slug-positive of stroma cells $\left(\chi^{2}=14.7, P=0.001\right)$.
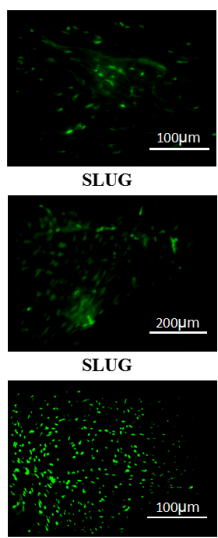

TWIST

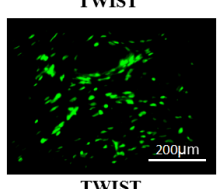

TWIST
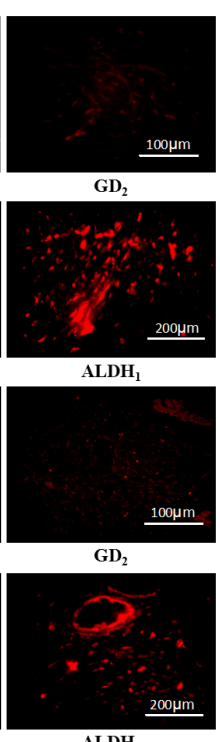
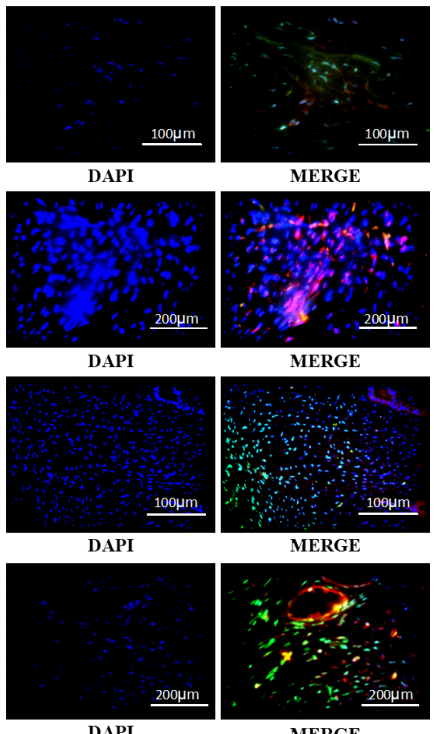

Figure 2: Representative photomicrographs of doubly stained immunofluorescence (IF) showing co-expression of EMT and MSCs markers in the malignant phyllodes tumor of the breast. The photomicrographs show SLUG paired with GD2 or ALDH1 and Twist with GD2 or ALDH1. 
Table 3: Comparison of the recurrence and metastasis, 5-year DFS and 5-year OS in three groups (benign, borderline and malignant phyllodes tumor of the breast) (Values of $P<0.05$ were considered significant)

\begin{tabular}{lccccc}
\hline Parameters & Benign & Borderline & Malignant & $\chi^{2}$ & $P$ values \\
\hline $\begin{array}{l}\text { Local recurrence } \\
\text { or distant }\end{array}$ & $14(13.5 \%)$ & $29(26.6 \%)$ & $14(42.4 \%)$ & 13.1 & 0.001 \\
metastasis* & & & & & \\
5-year DFS* & $99(95.2 \%)$ & $88(80.7 \%)$ & $22(66.7 \%)$ & 18.7 & $<0.001$ \\
5-year OS* & $101(97.1 \%)$ & $105(96.3 \%)$ & $26(78.8 \%)$ & 17.2 & $<0.001$ \\
\hline
\end{tabular}

$P$ values were calculated by Chi-square test.

* There were significant differences among benign, borderline and malignant phyllodes tumor of the breast in local recurrence or distant metastasis, 5-year DFS and 5-year OS $\left(\chi^{2}=13.1, P=0.001 ; \chi^{2}=18.7, P<0.001 ; \chi^{2}=17.2, P<0.001\right)$.

observed stronger E-cadherin expression in the epithelial compartment in comparison to the stroma. Among the 242 cases of PTs, high expression of E-cadherin was observed in the epithelial compartment of 132 cases $(64.4 \%$ of benign, $48.1 \%$ of borderline, and $31.1 \%$ of malignant cases), whereas, only 75 cases ( $31.7 \%$ of benign, $29.6 \%$ of borderline, and $33.3 \%$ of malignant) showed high expression in the stroma. In addition, high expression of E-cadherin in the epithelial compartment was more frequent in benign PTs compared with malignant and borderline PTs $(P=0.025)$. However, the E-cadherin expression was not different in the stroma of the different grades $(P=0.906)$. High expression of Snail with a nuclear staining pattern was observed in both the epithelial and the stromal cells of PTs tissues. The high expression of Snail in the epithelial and stromal cells was significantly different in three groups $(P=0.001$ and $P=0.007)$. Similarly, Slug and Twist showed high expression and a nuclear staining pattern in the epithelial and stromal cells of PTs with significant differences with progressive grades. Furthermore, Slug expression associated with the 5 -year-DFS and 5-year-OS of the patients.

MSCs play an important role in the progression of malignant tumors. Lin and colleagues demonstrated that $\mathrm{ALDH}_{1}$ and/or $\mathrm{GD}_{2}$ markers could be used for cancer stem cells research in malignant PTs [19]. We found coexpression of EMT and MSCs markers in $75 \%$ of the malignant cases (15/20 cases). Therefore, we hypothesized that malignant PTs possessed MSC characteristics and differentiated towards soft tissue tumor similar to liposarcoma, leiomyosarcoma, rhabdomyosarcoma, osteogenic sarcoma or chondrosarcoma. Further analysis with increased number of malignant PTs specimens is necessary to understand the significance of the coexpression and other statistics.

Surgery remains the only primary treatment in patients with PTs [28]. We evaluated survival outcomes of the 246 PTs patients, of which, 136 (55.3\%) were treated with Lumpectomy, 11 (4.5\%) with BCS (Breastconserving surgery), 29 (11.8\%) with mastectomy and 70 $(28.5 \%)$ with modified or radical mastectomy. Our studies found that the mode and extent of surgery associated with the 5-year-DFS $(P<0.01)$ and 5-year-OS $(P=0.002)$ rates. Previous studies had shown no significant differences in survival between patients that underwent BCS versus mastectomy and our data were partially concordant [29].

In conclusion, our study showed that expression of some EMT markers increased with the progression of tumor grade could be of prognostic value. We also observed that the mode of surgery, the grade of tumor and history of recent tumor growth were independent predictors for decreased OS. This study also indicated that PTs had a relatively milder prognosis compared to other epithelial tumors of the breast. The EMT signaling pathway and the mesenchymal stemness of PTs tumors present novel therapeutic targets in the future.
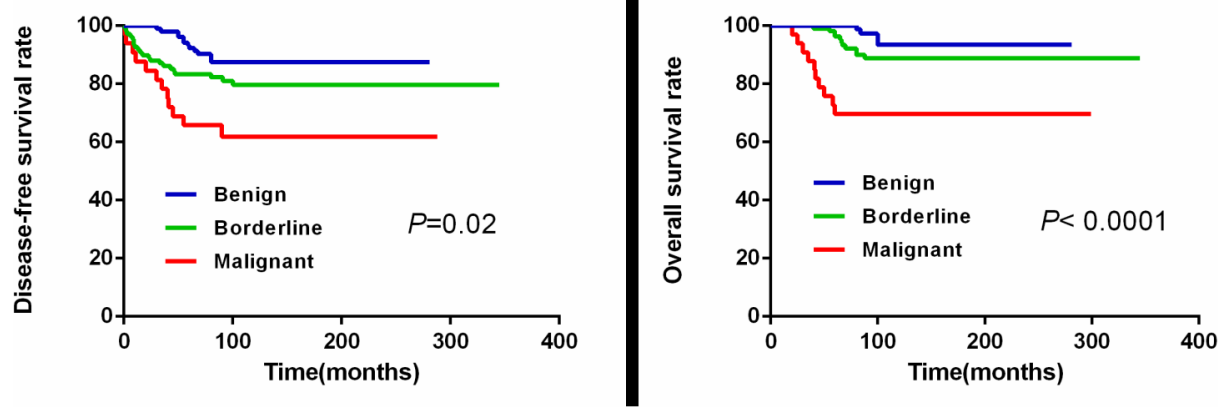

Figure 3: The 5-year DFS and 5-year OS curves in the three PTs patient groups. 
Table 4: Univariate analysis and multivariate analysis of malignant phyllodes tumor of the breast patients' survivals (Values of $\boldsymbol{P}<0.05$ were considered significant)

\begin{tabular}{|c|c|c|c|c|c|c|c|c|}
\hline \multirow{3}{*}{ Parameters } & \multicolumn{4}{|c|}{ DFS } & \multicolumn{4}{|c|}{ OS } \\
\hline & \multicolumn{2}{|c|}{ Univariate } & \multicolumn{2}{|c|}{ Multivariable } & \multicolumn{2}{|c|}{ Univariate } & \multicolumn{2}{|c|}{ Multivariable } \\
\hline & 5-year-DFS \% & $P$ & $95 \% \mathrm{CI}$ & $\boldsymbol{P}$ & 5-year-OS \% & $\boldsymbol{P}$ & $95 \% \mathrm{CI}$ & $\boldsymbol{P}$ \\
\hline \multicolumn{9}{|l|}{ Age } \\
\hline$\leq 50$ & $82.5 \%$ & \multirow{2}{*}{0.509} & & & $89.5 \%$ & \multirow{2}{*}{0.500} & & \\
\hline$>50$ & $81.1 \%$ & & & & $90.2 \%$ & & & \\
\hline \multicolumn{9}{|l|}{ Size } \\
\hline$\leq 3$ & $88.2 \%$ & \multirow{2}{*}{0.106} & & & $95.6 \%$ & \multirow{2}{*}{0.078} & & \\
\hline$>3$ & $79.1 \%$ & & & & $87.6 \%$ & & & \\
\hline \multicolumn{9}{|l|}{ Menopausal } \\
\hline Yes & $79.2 \%$ & \multirow{2}{*}{0.483} & & & $87.0 \%$ & \multirow{2}{*}{0.339} & & \\
\hline No & $82.2 \%$ & & & & $91.1 \%$ & & & \\
\hline Growth & & & & & & & $1.58-9.15$ & $0.003 *$ \\
\hline Yes & $83.6 \%$ & \multirow{2}{*}{0.135} & & & $80.7 \%$ & \multirow{2}{*}{$0.007^{*}$} & & \\
\hline No & $75.4 \%$ & & & & $92.6 \%$ & & & \\
\hline \multicolumn{9}{|l|}{ Painful } \\
\hline Yes & $83.6 \%$ & \multirow{2}{*}{0.137} & & & $86.0 \%$ & \multirow{2}{*}{0.374} & & \\
\hline No & $74 \%$ & & & & $90.8 \%$ & & & \\
\hline Grades & & & $0.90-2.23$ & 0.131 & & & $1.62-7.50$ & $0.001 *$ \\
\hline Benign & $88.5 \%$ & \multirow{3}{*}{$0.001 *$} & & & $96.2 \%$ & \multirow{3}{*}{$<0.001 *$} & & \\
\hline Borderline & $80.7 \%$ & & & & $89.9 \%$ & & & \\
\hline Malignant & $63.6 \%$ & & & & $69.7 \%$ & & & \\
\hline \multicolumn{9}{|l|}{ EMT marker } \\
\hline E-cadherin & $82.7 \%$ & 0.656 & & & $91.7 \%$ & 0.281 & & \\
\hline $\mathrm{N}$-cadherin & $81.5 \%$ & 0.925 & & & $90.3 \%$ & 0.830 & & \\
\hline Snail & $78.6 \%$ & 0.199 & & & $88.0 \%$ & 0.352 & & \\
\hline Slug & $76.6 \%$ & $0.036^{*}$ & $0.74-2.64$ & 0.304 & $85.5 \%$ & $0.028 *$ & $0.59-3.56$ & 0.421 \\
\hline Twist & $78.2 \%$ & 0.227 & & & $88.1 \%$ & 0.391 & & \\
\hline $\begin{array}{l}\text { Surgical } \\
\text { management }\end{array}$ & & & $1.08-1.74$ & $0.009 *$ & & & $0.80-1.57$ & 0.518 \\
\hline $\begin{array}{l}\text { Breast- } \\
\text { conserving } \\
\text { surgery }\end{array}$ & $100 \%$ & & & & $90.9 \%$ & & & \\
\hline Lumpectomy & $89.6 \%$ & \multirow{2}{*}{$<0.001 *$} & & & $95.6 \%$ & \multirow{2}{*}{$0.002 *$} & & \\
\hline Mastectomy & $64.3 \%$ & & & & $82.1 \%$ & & & \\
\hline $\begin{array}{l}\text { Modified/ } \\
\text { radical } \\
\text { mastectomy }\end{array}$ & $71.4 \%$ & & & & $82.9 \%$ & & & \\
\hline
\end{tabular}

DFS, disease-free survival; OS, overall survival. 


\section{MATERIALS AND METHODS}

\section{Patients and tissue samples}

This study was approved by the Tianjin Medical University Cancer Institute and Hospital, China and was performed in accordance with the ethical standards laid down by the 1964 Helsinki Declaration and its later amendments. After analyzing the clinicopathological parameters of breast cancer patients at the Cancer Hospital of Tianjin Medical University from April 1987 to June 2010, 246 patients ranging from 11 to 76 years old with a median age of 46 years were selected for our study. The inclusion criteria were: (a) The patients were diagnosed with PTs; (b) The patients had undergone surgery; (c) The patients had no previous neoadjuvant systemic therapy and (d) They completed the follow-up study period. Two senior pathologists reviewed the diagnosis and classification of all forms of PTs based on the 2012 WHO Classification of Tumors of the Breast by using hematoxylin and eosin (H\&E)-stained sections of samples from each selected case. The expression of EMT and mesenchymal stem cell biomarkers were evaluated for all 246 cases by performing IHC. To study the clinical relevance of EMT biomarkers expression, the 246 cases were divided into three groups (104 benign cases, 109 borderline cases, and 33 malignant cases) according to their tumor grade.

\section{Pathological review}

All cases were reviewed by two senior pathologists (Niu Y. and Lv SH.). Tumors were evaluated for stromal cellularity and stromal nuclear atypia [30]. The stromal cellularity was classified as (a) low : if the stromal cells were sparsely distributed and appeared as slender spindle cells; (b) moderate : if the cellularity was moderate and the stromal cells were similar to spindle cells with slight pleomorphism and (c) high, if the cellularity was high with the stromal cells showing back-to-back arrangement with malignant appearance and nuclear pleomorphism. When the cellularity within an individual tumor was heterogeneous and showed other sarcoma of soft connective, the higher score was recorded (Figure 1). The stromal mitotic activity was quantified based on ten highpower fields (HPFs) in the most mitotically active areas of the stroma. Heterologous differentiation components such as rhabdomyosarcoma, chondrosarcoma and osteosarcoma were screened by hematoxylin-eosin staining and confirmed by additional immunohistochemical stains such as Myogenin, Myo D1, Desmin and S-100 protein.

According to 2012 WHO Classification of Tumors of the Breast [31], the histopathological grade of PTs was determined as low, moderate or high grade cellularity with nuclear atypia and classified as benign, borderline and malignant, respectively. Moreover, cellularity and/ or mitotic counts were also considered. The 2012 WHO
Classification of Tumors of the Breast proposed a cutoff of 5 mitoses per 10 HPFs to distinguish between benign and borderline PT. Moderately atypical cases with both $\geq$ 10 mitoses/10HPFs and diffusely high cellularity were upgraded to malignant. In summary, malignant, borderline and benign PTs showed $\geq 10, \leq 9$ and $\leq 4$ mitoses per $10 \mathrm{HPFs}$, respectively.

\section{Immunohistochemical analysis}

The tissue samples were fixed in $10 \%$ neutral buffered formalin and then paraffin-embedded. Then, $3 \mu \mathrm{m}$ sections were cut and de-waxed on coated slides with xylene followed by rehydration with distilled water. Further, sections were treated with citrate buffer solution ( $\mathrm{pH}$ 6.0) or ethylene diamine tetraacetic acid (EDTA) buffer (pH 8.0) for $2.5 \mathrm{~min}$ in a pressure cooker for antigen retrieval. This was followed by treatment with 3\% hydrogen peroxide and blocking in normal goat serum for $20 \mathrm{~min}$. The primary antibodies that were used in this study were: E-cadherin (ZSGB, ZM-0565, CHN), N-cadherin (ZSGB, ZM-0094, CHN), Vimentin (ZSGB, ZM-0511, CHN), Snail (Abcam, ab180714, UK), Slug (Abcam, ab27568, UK), Twist (Abcam, ab50581, UK), GD (Abcam, ab68456, UK) and $\mathrm{ALDH}_{1}$ (Abcam, ab56777, UK). They were used at dilutions of 1:150, 1:50, $1: 150,1: 100,1: 100,1: 100,1: 100$ and 1:70, respectively. After incubation with primary antibodies at $4^{\circ} \mathrm{C}$ overnight, the secondary biotinylated goat anti-mouse/anti-rabbit immunoglobulin was applied for $20 \mathrm{~min}$ followed by incubation with peroxidase-conjugated streptavidin. Then, sections were incubated with 3, 3'-diaminobenzidine tetrahydrochloride (DAB) and counterstained with hematoxylin. Sections of normal breast epithelial cells and external tissues were processed simultaneously and served as positive controls. For negative controls, primary antibodies were replaced by PBS. The stromal and epithelial cells of the tumors were scored separately based on IHC staining by two senior pathologists (Niu Y. and Lv SH.) based on staining intensity (negative, weak, mild or strong), percentage of positive cells and the cellular localization of staining (nucleus, cytoplasmic or membranous) of these slides.

\section{Immunofluorescence staining}

The paraffin sections of malignant PTs were dewaxed in xylene and rehydrated followed by antigen retrieval in ethylene diamine tetraacetic acid (EDTA) buffer (pH 8.0) by pressure cooking for $2 \mathrm{~min}$. Subsequently, the sections were permeabilized with $0.1 \%$ Triton X-100 and incubated overnight at $4^{\circ} \mathrm{C}$ with rabbit anti-human Snail (Abcam, ab180714, UK), Slug (Abcam, ab180714, UK), Twist (Abcam, ab50581, UK), mouse anti-human GD (Abcam, ab68456, UK) and ALDH1 (Abcam, ab56777, UK) antibodies. Then, the sections 
were rinsed and incubated with the secondary antibody conjugated to the fluorescent dyes, Alexa Fluor ${ }^{\circledR} 488$ and Alexa Fluor ${ }^{\circledR}$ 594. Finally, the sections were rinsed and incubated with 4', 6-diamidino-2-phenylindole dihydrochloride (DAPI; Life Technologies, USA) and visualized under a fluorescence microscope.

\section{Follow-up study}

The cases were followed-up until March 1, 2016 and ranged from 60 to 345 months with a median duration of 102.5 months. Detailed dates of follow-up cases were collected via medical records, telephone calls and a study questionnaire every 3 months for the first 2 years and every 6 months for the next 3 years. Disease-free survival (DFS) was defined from the date of surgery to the date of local relapse and/or distant metastasis. Reappearance of tumor in the treated remnant breast, skin or chest wall was defined as local recurrence. Distant metastasis was considered when the tumor metastasized at a distant site like lungs, bone and/or liver.

\section{Statistical analysis}

Statistical analyses were performed using SPSS 19.0 software (SPSS Inc., Chicago, IL, USA). Univariate $\chi^{2}$ tests were used to investigate associations between tumor characteristics and biomarker positivity. When necessary, fisher's exact test was used. Survival analysis was performed by using the Kaplan-Meier curves and log-rank tests. Cox proportional hazards modeling for multivariate analysis was used to identify variables of independent prognostic value. Results were considered significant if a $P$ value of $<0.05$ was obtained.

\section{ACKNOWLEDGMENTS}

This work was financially supported by National Natural Science Foundation of China (811470119).

\section{CONFLICTS OF INTEREST}

The authors declare that they have no conflicts of interest.

\section{REFERENCES}

1. Belkacemi Y, Bousquet G, Marsiglia H, Ray-Coquard I, Magne N, Malard Y, Lacroix M, Gutierrez C, Senkus E, Christie D, Drumea K, Lagneau E, Kadish SP, et al. Phyllodes tumor of the breast. Int J Radiat Oncol Biol Phys. 2008; 70:492-500. doi: 10.1016/j.ijrobp.2007.06.059.

2. Wang F, Jia Y, Tong Z. Comparison of the clinical and prognostic features of primary breast sarcomas and malignant phyllodes tumor. Jpn J Clin Oncol. 2015; 45:14652. doi: 10.1093/jjco/hyu177.
3. Vergine M, Guy C, Taylor MR. Phyllodes Tumor of the Breast With Malignant Melanoma Component: A Case Report. Int J Surg Pathol. 2015; 23:483-8. doi: 10.1177/1066896915592018.

4. Pfarr N, Kriegsmann M, Sinn P, Klauschen F, Endris V, Herpel E, Muckenhuber A, Jesinghaus M, Klosterhalfen B, Penzel R, Lennerz JK, Weichert W, Stenzinger A. Distribution of MED12 mutations in fibroadenomas and phyllodes tumors of the breast--implications for tumor biology and pathological diagnosis. Genes Chromosomes Cancer. 2015; 54:444-52. doi: 10.1002/gcc.22256.

5. Katsohis CD, Fahandides E, Agurigakis C, Aletras HA. Cystosarcoma phyllodes of the breast. Int Surg. 1990; 75:162-5.

6. Liu J, Liu X, Feng X, Liu J, Lv S, Zhang W, Niu Y. C-kit overexpression correlates with KIT gene copy numbers increases in phyllodes tumors of the breast. Breast Cancer Res Treat. 2015; 149:395-401. doi: 10.1007/ s10549-014-3214-1.

7. Mitus JW, Blecharz P, Walasek T, Reinfuss M, Jakubowicz J, Kulpa J. Treatment of Patients with Distant Metastases from Phyllodes Tumor of the Breast. World J Surg. 2016; 40:323-8. doi: 10.1007/s00268-015-3262-7.

8. Kalluri R, Weinberg RA. The basics of epithelialmesenchymal transition. J Clin Invest. 2009; 119:1420-8. doi: 10.1172/JCI39104.

9. Zhu X, Zhong J, Zhao Z, Sheng J, Wang J, Liu J, Cui K, Chang J, Zhao H, Wong S. Epithelial derived CTGF promotes breast tumor progression via inducing EMT and collagen I fibers deposition. Oncotarget. 2015; 6:25320-38. doi: 10.18632/oncotarget.4659.

10. Parvani JG, Gujrati MD, Mack MA, Schiemann WP, Lu ZR. Silencing beta3 Integrin by Targeted ECO/siRNA Nanoparticles Inhibits EMT and Metastasis of TripleNegative Breast Cancer. Cancer Res. 2015; 75:2316-25. doi: 10.1158/0008-5472.CAN-14-3485.

11. Pang MF, Georgoudaki AM, Lambut L, Johansson J, Tabor V, Hagikura K, Jin Y, Jansson M, Alexander JS, Nelson CM, Jakobsson L, Betsholtz C, Sund M, et al. TGF-beta1induced EMT promotes targeted migration of breast cancer cells through the lymphatic system by the activation of CCR7/CCL21-mediated chemotaxis. Oncogene. 2015; 35:748-60. doi: 10.1038/onc.2015.133.

12. Biswas S, Sengupta S, Roy Chowdhury S, Jana S, Mandal G, Mandal PK, Saha N, Malhotra V, Gupta A, Kuprash DV, Bhattacharyya A. Erratum to: CXCL13-CXCR5 co-expression regulates epithelial to mesenchymal transition of breast cancer cells during lymph node metastasis. Breast Cancer Res Treat. 2016; 155:615-6. doi: 10.1007/ s10549-016-3713-3.

13. Gao T, Li JZ, Lu Y, Zhang CY, Li Q, Mao J, Li LH. The mechanism between epithelial mesenchymal transition in breast cancer and hypoxia microenvironment. Biomed Pharmacother. 2016; 80:393-405. doi: 10.1016/j. biopha.2016.02.044. 
14. Hyun KA, Goo KB, Han H, Sohn J, Choi W, Kim SI, Jung HI, Kim YS. Epithelial-to-mesenchymal transition leads to loss of EpCAM and different physical properties in circulating tumor cells from metastatic breast cancer. Oncotarget. 2016; 7:24677-87. doi: 10.18632/oncotarget.8250.

15. Sun L, Burnett J, Gasparyan M, Xu F, Jiang H, Lin CC, Myers I, Korkaya H, Liu Y, Connarn J, He H, Zhang N, Wicha MS, et al. Novel cancer stem cell targets during epithelial to mesenchymal transition in PTEN-deficient trastuzumab-resistant breast cancer. Oncotarget. 2016; 7:51408-51422. doi:10.18632/oncotarget.9839.

16. Tan X, Fu Y, Chen L, Lee W, Lai Y, Rezaei K, Tabbara S, Latham P, Teal CB, Man YG, Siegel RS, Brem RF, Fu SW. miR-671-5p inhibits epithelial-to-mesenchymal transition by downregulating FOXM1 expression in breast cancer. Oncotarget. 2016; 7:293-307. doi: 10.18632/ oncotarget.6344.

17. Xu H, Li M, Zhou Y, Wang F, Li X, Wang L, Fan Q. S100A4 participates in epithelial-mesenchymal transition in breast cancer via targeting MMP2. Tumour Biol. 2016; 37:2925-32. doi: 10.1007/s13277-015-3709-3.

18. Yin K, Yin W, Wang Y, Zhou L, Liu Y, Yang G, Wang J, Lu J. MiR-206 suppresses epithelial mesenchymal transition by targeting TGF-beta signaling in estrogen receptor positive breast cancer cells. Oncotarget. 2016; 7:24537-48. doi: 10.18632/oncotarget.8233.

19. Lin JJ, Huang CS, Yu J, Liao GS, Lien HC, Hung JT, Lin RJ, Chou FP, Yeh KT, Yu AL. Malignant phyllodes tumors display mesenchymal stem cell features and aldehyde dehydrogenase/disialoganglioside identify their tumor stem cells. Breast Cancer Res. 2014; 16:R29. doi: 10.1186/ bcr3631.

20. Takizawa K, Yamamoto H, Taguchi K, Ohno S, Tokunaga E, Yamashita N, Kubo M, Nakamura M, Oda Y. Insulinlike growth factor II messenger RNA-binding protein-3 is an indicator of malignant phyllodes tumor of the breast. Human Pathology. 2016; 55:30-8. doi: 10.1016/j. humpath.2016.04.007.

21. Vorherr H, Vorherr UF, Kutvirt DM, Key CR. Cystosarcoma phyllodes: epidemiology, pathohistology, pathobiology, diagnosis, therapy, and survival. Arch Gynecol. 1985; 236:173-81.

22. Lim JC, Koh VC, Tan JS, Tan WJ, Thike AA, Tan PH. Prognostic significance of epithelial-mesenchymal transition proteins Twist and Foxc2 in phyllodes tumours of the breast. Breast Cancer Res Treat. 2015; 150:19-29. doi: 10.1007/s10549-015-3296-4.

23. Kwon JE, Jung WH, Koo JS. Molecules involved in epithelial-mesenchymal transition and epithelial-stromal interaction in phyllodes tumors: implications for histologic grade and prognosis. Tumour Biol. 2012; 33:787-98. doi: 10.1007/s13277-011-0296-9.

24. Zhou Z, Wang S, Song C, Hu Z. Paeoniflorin prevents hypoxia-induced epithelial-mesenchymal transition in human breast cancer cells. Onco Targets Ther. 2016; 9:2511-8. doi: 10.2147/OTT.S102422.

25. Zhao Z, Sun YS, Chen W, Lv LX, Li YQ. Hispolon inhibits breast cancer cell migration by reversal of epithelial-tomesenchymal transition via suppressing the ROS/ERK/ Slug/E-cadherin pathway. Oncol Rep. 2016; 35:896-904. doi: 10.3892/or.2015.4445.

26. Zhang P, Yang X, Yin Q, Yi J, Shen W, Zhao L, Zhu Z, Liu J. Inhibition of SK4 Potassium Channels Suppresses Cell Proliferation, Migration and the Epithelial-Mesenchymal Transition in Triple-Negative Breast Cancer Cells. PLoS One. 2016; 11:e0154471. doi: 10.1371/journal. pone. 0154471 .

27. Do SI, Kim JY, Kang SY, Lee JJ, Lee JE, Nam SJ, Cho EY. Expression of TWIST1, Snail, Slug, and NF-kappaB and methylation of the TWIST1 promoter in mammary phyllodes tumor. Tumour Biol. 2013; 34:445-53. doi: 10.1007/s13277-012-0569-y.

28. Tse GM, Niu Y, Shi HJ. Phyllodes tumor of the breast: an update. Breast Cancer. 2010; 17:29-34. doi: 10.1007/ s12282-009-0114-z.

29. Bernardi G, Cavallaro G, Indinnimeo M, Fiore A, Basso L, D'Ermo G, De Toma G, Cavallaro A. Usefulness of ultrasounds in the management of breast phyllodes tumors. G Chir. 2012; 33:81-5.

30. Mituś J, Reinfuss M, Mituś JW, Jakubowicz J, Blecharz P, Wysocki WM, Skotnicki P. Malignant Phyllodes Tumor of the Breast: Treatment and Prognosis. The Breast Journal. 2014; 20:639-44. doi: 10.1111/tbj.12333.

31. Lakhani SR, Ellis IO, Schnitt SJ, Tan PH, Van der Vijver MJ. (2012). WHO classification of tumours of the breast. WHO, Geneva. 